\title{
Tendência da incidência de HIV-aids segundo diferentes critérios diagnósticos em Campinas-SP, Brasil de 1980 a 2016
}

\author{
Trend incidence of HIV-AIDS according to different diagnostic \\ criteria in Campinas-SP, Brazil from 1980 to 2016
}

Márcio Cristiano de Melo (https://orcid.org/0000-0001-9840-0309) ${ }^{1}$

Valéria Correia de Almeida (https://orcid.org/0000-0002-4027-7835) ${ }^{2}$

Maria Rita Donalísio (https://orcid.org/0000-0003-4457-9897) ${ }^{1}$
${ }^{1}$ Departamento de Saúde Coletiva, Universidade Estadual de Campinas. R. Tessália Vieira de Camargo 126, Cidade Universitária Zeferino Vaz. 13083-887 Campinas SP Brasil. enf.marciomelo@gmail.com ${ }^{2}$ Departamento de Vigilância em Saúde, Secretaria Municipal de Saúde de Campinas. Campinas SP Brasil.

\begin{abstract}
The objective of this study was to analyze the temporal trend of the incidence coefficients of HIV-AIDS infection via a retrospective ecological study and segmented regression in individuals older than 13 year reported at SINAN between 1980 and 2016. Of the 14,204 reported cases, $70.9 \%$ were male and $39.5 \%$ were white. The AIDS criterion presented growing incidence from 1986 to 1996 with Annual Percentual Change (APC) of 108.5\% per year between 1983 and 1989 (CI: 90.3 - 128.4), between 1989 and 1998 the APC was $13.2 \%$ (CI: $8.2-18.3$ ) and APC reduction in the period from 1998 to 2016, -6.2\% (CI: $-7.4-4.9)$. High coefficients of the death criteria between 1988 and 2002 (4.7/100,000 inhabitants), with a noticeable decrease after 1995. The growth of the HIV+ criteria from 2000 onward was observed, reaching 30.2 cases per 100,000 inhabitants in 2016. Although incidence rates still remain high in the municipality, these trends are consistent with the positive impact of early detection policies and access to clinical and therapeutic follow-up of individuals with HIV and AIDS in Campinas.
\end{abstract}

Key words Incidence, AIDS, HIV, Disease Notification, Health Information Systems
Resumo $O$ estudo teve por objetivo analisar a tendência temporal dos coeficientes de incidência da infecção por HIV-aids por meio de estudo ecológico retrospectivo e regressão segmentada em maiores de 13 anos notificados no SINAN entre os anos de 1980 e 2016. Dos 14.204 casos notificados, $70,9 \%$ eram do sexo masculino e $39,5 \%$ brancos. Nota-se aumento da incidencia do critério aids de 1986 a 1996 com variação anual percentual (APC) de 108,5\% ao ano entre 1983 a 1989 (IC: 90,3 - 128,4), entre os anos de 1989 e 1998 a APC foi de 13,2\% (IC: 8,2 - 18,3) e redução da APC no período de 1998 a 2016, -6,2\% (IC: -7,4 - -4,9). Altos coeficientes do critério óbito entre os anos 1988 a 2002 (4,7/100.00 hab.) com queda acentuada após 1995. Observa-se crescimento do critério HIV+ a partir do ano 2000, chegando a 30,2 por 100 mil habitantes em 2016. Embora as taxas de incidência ainda permaneçam altas no município, estas tendências são compatíveis com o impacto positivo das políticas de detecção precoce e acesso ao seguimento clínico e terapêutico dos indivíduos com HIV e aids na cidade.

Palavras-chave Incidência, Aids, HIV, Notificação de Doenças, Sistemas de Informação em Saúde 


\section{Introdução}

A aids é uma doença de notificação compulsória no Brasil desde 1986 de forma descentralizada, registrada sistematicamente no Sistema de Informação de Agravos de Notificação (SINAN), (Portaria MS no 542, 1986) com mudanças nos códigos de notificação da doença ao longo do tempo. Até 31 de março de 2003 considerava-se casos de aids de acordo com o critério Centers for Disease Control (CDC) (código 100), CDC/ Laboratório (código 200), Rio de Janeiro (RJ)/ Caracas (código 300), CDC + CDC/Laboratório (código 120), CDC + RJ/Caracas (código 130), CDC/Laboratório + RJ/Caracas (código 140), $\mathrm{CDC}+\mathrm{CDC} /$ Laboratório + RJ/Caracas (código 123), CDC Excepcional (código 400), AIDS Related Complex (ARC) + Óbito (código 500), Óbito (código 600) ) $^{1,2}$.

A partir desta data, os principais critérios foram revistos, mantendo-se o RJ/Caracas e introduzindo adaptações e ajustes no critério CDC Modificado, sendo denominado como CDC Adaptado. Com relação aos demais critérios, foram excluídos o ARC + Óbito e o CDC Excepcional, permanecendo o critério Óbito que incorporou não apenas a menção de aids e de seus termos correlatos em algum campo da Declaração de Óbito (DO), mas também a menção de infecção pelo HIV ou termos semelhantes. Tais variáveis são preenchidas no SINAN, segundo os critérios descritos acima, seguindo uma hierarquização por ordem de importância (maior para o menor): 100, 300, 600 e 901 . As categorias 200 , $120,140,123,400$ e 500 foram excluídas a partir da versão 5.0 do SINAN, mantendo critérios de mudança e hierarquização dos casos ${ }^{1}$.

O estado de São Paulo demonstrou desde a década de 1990 a intenção de trabalhar com a notificação dos portadores assintomáticos do HIV, entendendo que o perfil epidemiológico destes reflete um padrão mais recente que os casos de aids. Em 1994 o Programa Estadual de DST/Aids propôs a notificação voluntária dos casos de HIV no estado através do Sistema de Informação de Soropositivo Assintomático (SIHIV) ${ }^{3}$. A partir de 2000 passou a ser utilizado o SINAN como ferramenta de transmissão de dados, sendo a adoção dessa medida diferenciada entre os municípios ${ }^{4} \mathrm{O}$ que propiciou a obtenção de estimativas do número de infecções pelo HIV, de casos de aids e de mortes. Tais dados permitem a monitoração espaço-temporal da doença no país e a identificação de desigualdades regionais na incidência e mortalidade associada à aids.
A Vigilância da doença no Brasil inclui desde a identificação da possibilidade de cruzamento de bancos de dados (Reck-link). Além de dados do SINAN, podem ser utilizados dados obtidos a partir do Sistema de Informação sobre Mortalidade (SIM), do Sistema de Controle de Exames Laboratoriais da Rede Nacional de Contagem de Linfócitos CD4+/CD8+ e Carga Viral do HIV (SISCEL), do Sistema de Controle Logístico de Medicamentos (SICLOM), que são sistemas específicos pra controle de exames e medicamentos em pessoas vivendo com HIV/aids (PVHA). Tais sistemas têm sido utilizados para análises de variáveis sociodemográficas, comportamentais, epidemiológicas e de utilização de serviços de saúde, e permitem ainda o monitoramento de fatores determinantes da infecção e seu potencial de disseminação em grupos populacionais específicos, subsidiando ações de prevenção e controle ${ }^{5,6}$.

Estudos têm demonstrado que o diagnóstico precoce da infecção por HIV, medidas profiláticas, o seguimento clínico e a adesão ao tratamento com antirretrovirais influenciam diretamente a sobrevida dos pacientes ${ }^{7,8}$. Por outro lado, os coeficientes de incidência de HIV no Brasil permanecem altos na última década ${ }^{9,10}$.

O perfil epidemiológico da infecção por HIV e aids modificou-se (deixou de ser majoritariamente concentrado em grandes centros urbanos com migração para o interior) no decorrer das décadas no Brasil, com queda nos coeficientes de mortalidade e aumento da detecção de casos, porém com diferenças regionais distintas. No final da década de 2000 observaram-se mudanças na tendência da epidemia levando em consideração as novas políticas assistenciais brasileiras ${ }^{11}$.

Campinas é uma grande cidade do interior do estado de São Paulo que concentra a segunda maior prevalência de HIV/aids do estado em 2017 $7^{12}$. Possui o Programa Municipal DST-Aids implantado desde 1993, e, juntamente com o centro de referência em aids do Hospital das Clínicas da Universidade Estadual de Campinas (Unicamp), atendem aproximadamente $80 \%$ da demanda do município. O Programa municipal tem implantado várias ações de prevenção $\mathrm{e}$ detecção precoce de HIV nas últimas décadas e um sistema de vigilância atuante desde o início da epidemia ${ }^{13}$ priorizando ações educativas, preventivas e assistenciais, de forma descentralizada, integrada e humanizada, em parceria com a sociedade civil.

Foram analisados os coeficientes de incidência de HIV e aids com a finalidade de observar a progressão da doença no contexto do município 
de Campinas a fim de prevenir agravos como: aumento no número de casos, coinfecções e falhas na prevenção, tratamento e segmento. O objetivo deste trabalho é analisar a tendência temporal dos coeficientes de incidência da infecção por HIV-aids em maiores de 13 anos em Campinas de 1980 a 2016, de acordo com os critérios de notificação adotados pelo Ministério da Saúde (MS), infecção por HIV, aids e óbito, e variáveis sociodemográficas e epidemiológicas.

\section{Método}

Foi estudada a totalidade dos casos registrados no SINAN de 1980 a 2016 disponibilizados pelo Departamento de Vigilância em Saúde (DEVISA) da cidade de Campinas. Trata-se de um estudo ecológico da tendência temporal dos coeficientes de incidência de aids por 100 mil habitantes em maiores de 13 anos em Campinas. Este município apresenta estimativa populacional de 1.100 .000 habitantes, está situado a nordeste do estado de São Paulo, é um polo industrial, tecnológico e centro de referência médico-hospitalar da região.

Como critério de inclusão no estudo, utilizou-se a faixa etária de maiores de 13 anos e o município de residência do indivíduo ser Campinas. Foram analisadas as seguintes variáveis constantes na ficha de notificação epidemiológica: sexo (masculino e feminino); raça/cor autodeclarada (branca, preta, amarela, parda, indígena e ignorada); escolaridade em anos de estudos concluídos (nenhuma, 1 a 3, 4 a 7, 8 a 11, 12 ou mais e ignorada); prática sexual (homens que fazem sexo com homens (HSH), mulheres que fazem sexo com mulheres (MSM), heterossexual, bissexual, não se aplica e ignorada); exposição sanguínea ( $\operatorname{sim}$, não e ignorada) e critério diagnóstico (aids, óbito e $H I V+)$.

\section{Etapa 1: Cálculo de estimativas populacionais para o período de 2013 a 2016}

Para os anos de 2013 a 2016 foram calculadas as estimativas populacionais adotando o crescimento aritmético, uma vez que tal dado não constava nos bancos oficiais brasileiros.

Considerou-se que a população evoluiu segundo um incremento constante por unidade de tempo, no decorrer do período entre dois censos oficiais (ano a ano, o crescimento é constante). Para tal, calculou-se a média das populações entre os dois censos evidenciada pelo Crescimento Médio Anual (CMA) $\left(\mathrm{P}_{1}-\mathrm{P}_{0} / \mathrm{t}_{1}-\mathrm{t}_{0}\right.$, onde: $\mathrm{t}_{0}=$ ano do $1^{\circ}$ censo; $\mathrm{t}_{1}=$ ano do $2^{\circ}$ censo; $\mathrm{P}_{0}=$ população do $1^{\circ}$ censo; $\mathrm{P}_{1}=$ população do $2^{\circ}$ censo). Admitindo-se a constância desse crescimento anual, para estimar uma dada população $\mathrm{P}_{\mathrm{x}}$ em um ano $\mathrm{t}_{\mathrm{x}}$, utilizou-se a expressão $\left(\mathrm{P}_{\mathrm{x}}=\mathrm{P}_{0}\right.$ $+\left[\right.$ CMA $\left.\left.\left(t_{x}-t_{0}\right)\right]\right)\left(\right.$ onde $t_{x}-t_{0}=$ tempo decorrido entre a data do $1^{\circ}$ censo e o ano de que se quer conhecer a população $)^{14}$.

Para o cálculo de coeficientes, há a necessidade de conhecer a população para o meio do ano, isto é, $1^{\circ}$ de julho, porém o censo é dado para $1^{\circ}$ de setembro. Portanto, para conhecer a população relativa de cada ano sem informação, admitiu-se que o CMA é constante, da mesma forma adotou-se que o Crescimento Médio Mensal (CMM) também é constante e, na unidade de tempo (mês) será 1/12 do CMA, isto é, CMA/12. Para obter a população de $1^{\circ}$ de julho aceita-se a expressão: $\left[\mathrm{P}_{1 / \text { jul/ano }}=\mathrm{P}_{1 / \text { set/ano }}-(2 \times \mathrm{CMM})\right]$ (onde $\mathrm{P}=$ população $)^{14}$.

\section{Etapa 2: Estimativas da variação percentual anual}

Para a aplicabilidade do método proposto, realizaram-se testes para averiguar se os dados de incidência durante a série temporal estudada possuíam pressuposição de confiabilidade para o uso da regressão segmentada. Foram verificadas: $1^{\circ}$ ) a homocedasticidade (para designar variância constante das incidências para observações temporais distintas); 2o) a autocorrelação (quando não há relação entre valores ordenados segundo tempo ou espaço); 3º a normalidade dos resíduos dos modelos mais adequados para os ajustes (quando os dados seguem distribuição normal). Para tais análises foi utilizado o software Stata 11.

Foi estimada a variação anual percentual (Annual Percentual Change, APC), que demonstra variações positivas ou negativas da incidência durante uma série temporal, por meio de uma regressão segmentada (RS) verificando pontos importantes de mudança. São ajustados sucessivos segmentos da reta conectados entre si por esses pontos de mudança. O número de pontos necessários para o ajuste de cada segmento foi selecionado automaticamente pela configuração padrão do programa (sem a transformação logarítmica da variável resposta) Joinpoint Regression Program versão 4.5.0.1, fornecido pelo Instituto Nacional de Câncer dos Estados Unidos (Nacional Cancer Institute) ${ }^{15,16}$. Foram calculados intervalos de confiança de $95 \%$ para a APC.

O software utiliza dados de tendência que se encaixam em um modelo (gráfico) de pontos li- 
gados (joinpoint) mais simples que os dados de um gráfico de incidência normal permitindo testar se uma mudança aparente na tendência é estatisticamente significativa. Os testes de significância utilizam um método de Permutação de Monte Carlo, podendo incorporar variação estimada para cada ponto ou usar um modelo de variação de Poisson. Além disso, os modelos também podem ser lineares no log da resposta, o que permite o cálculo da $\mathrm{APC}^{17}$.

Foram estimadas as variações percentuais anuais obtidas na regressão segmentada (Joinpoint Regression) com os respectivos intervalos de confiança de $95 \%$ e teste qui-quadrado com correção de Yates (considerando 5\% de significância para os testes estatísticos).

As curvas de incidência de casos pelo critério óbito e infecção pelo HIV não foram analisados pelo Joinpoint Regression devido a não se adequarem aos critérios de homocedasticidade e normalidade.

\section{Etapa 3: Análise dos coeficientes de incidência}

Para a comparação entre os códigos de notificação foram analisados os códigos 100, 200, 300, 120, 130, 140, 123 e 400 (critério de definição de aids), código 600 (óbito) e código 901 (HIV+). Para o critério aids considerou-se todos os casos em indivíduos com mais de 13 anos registrados com qualquer código definidor da doença ${ }^{18}$.

Os coeficientes de detecção de casos novos ou coeficientes de incidência foram calculados utilizando-se casos notificados segundo cada critério diagnósticos ("aids", "HIV" e "óbito") como numerador e as populações dos anos dos censos e respectivas estimativas populacionais, como denominadores para cada 100 mil habitantes.

Os dados foram apresentados em forma de tabelas e gráficos com as tendências dos coeficientes de incidência, com suavização por médias móveis (SMM), onde cada observação na série suavizada representa a média de três pontos adjacentes da série original. Tal análise remove variações aleatórias e mostra os componentes de tendência e ciclo ponderando todas as observações passadas igualmente. Para tanto, foi utilizado o software Excel 2013 para Windows.

A distribuição percentual das notificações segundo variáveis de interesse e critérios diagnósticos não foram comparadas devido ao número elevado de categorias "ignorado" em algumas variáveis. Tais valores, por serem altos, inviabilizam uma análise comparativa.
O projeto foi aprovado pelo Comitê de Ética de Pesquisas em Seres Humanos, da Faculdade de Ciências Médicas da Unicamp.

\section{Resultados}

Em Campinas, no período de 1980 a 2016, foram notificados 14.204 casos de HIV/aids em maiores de 13 anos entre todos os critérios de notificação, o que equivale a 5,5\% dos casos notificados no estado de São Paulo no mesmo período ( $\mathrm{n}=$ 258.696) de acordo com o Boletim Epidemiológico - Aids e DST de 2016², sendo 76,6\% casos de aids (todos os critérios) $(\mathrm{n}=10.876)$.

A Tabela 1 mostra a distribuição percentual dos casos de HIV/aids de acordo com os critérios de notificação da doença e as variáveis sociodemográficas e epidemiológicas. Ao se comparar a distribuição das notificações entre os sexos, verificou-se que a maior proporção dos casos é do critério aids, 76,4\% entre as mulheres e 77,1\% entre os homens. A média de idade entre os homens foi de 35,2 anos e mediana de 33,8 anos, entre as mulheres foi de 35,1 anos e 33,3 anos, respectivamente (dado não apresentado em tabela).

Observa-se maior concentração dos casos na raça/cor branca $(39,5 \%)$, considerando todos os critérios diagnósticos, com escolaridade entre quatro e sete anos $(13,7 \%)$. Com relação às faixas etárias, predominam-se pessoas entre 30 a 39 anos (38,9\%). Quanto à prática sexual, 58,1\% foram notificadas como heterossexuais e $20,9 \%$ como HSH, destes, $58,0 \%$ e $21,2 \%$ foram notificados com aids (todos os critérios) respectivamente. Apresentaram exposição sanguínea ao vírus (considerando utilização de drogas injetáveis (UDI), antecedente de hemofilia, transfusão sanguínea e acidente com material biológico) 19,1\% dos indivíduos (Tabela 1).

Na comparação dos coeficientes de incidência entre os sexos e os critérios diagnósticos em Campinas, nota-se um aumento da incidência de aids (todos os critérios) por 100 mil habitantes a partir do ano de 1986 até o ano de 1996 (Figura 1A). Destacam-se altos coeficientes do critério óbito, particularmente entre os anos de 1988 a 2002, chegando a 4,7 casos notificados por esse critério por 100 mil habitantes, com queda acentuada após 1995, verificada pela tendência decrescente da curva suavizada (Figura 1B). Observa-se um crescimento considerável do critério notificação HIV+ a partir do ano 2000, evidenciado pela curva suavizada, chegando a 30,2 por 100 mil habitantes no final do período (Figura 1C). 
Tabela 1. Percentual de casos de HIV/aids em maiores de 13 anos de acordo com o critério diagnóstico e variáveis sociodemográficas e epidemiológicas em Campinas-SP, Brasil, 1980-2016.

\begin{tabular}{|c|c|c|c|c|c|c|c|c|c|c|}
\hline & & \multicolumn{2}{|c|}{ Aids (CDC) } & \multicolumn{2}{|c|}{ Óbito } & \multicolumn{2}{|c|}{ HIV+ } & \multicolumn{2}{|c|}{ Total $^{* * *}$} & \multirow{2}{*}{ p-valor } \\
\hline & & $\mathbf{N}$ & $\%$ & $\mathbf{N}$ & $\%$ & $\mathbf{N}$ & $\%$ & $\mathbf{N}$ & $\%$ & \\
\hline \multirow[t]{2}{*}{ Sexo } & Feminino & 3185 & 76,4 & 54 & 2,3 & 891 & 21,4 & 4132 & 100,0 & 0,001 \\
\hline & Masculino & 7691 & 77,1 & 230 & 1,3 & 2151 & 21,6 & 10072 & 100,0 & \\
\hline \multirow[t]{6}{*}{ Raça/Cor } & Branca & 3970 & 70,7 & 9 & 0,2 & 1634 & 29,1 & 5613 & 100,0 & 0,000 \\
\hline & Preta & 563 & 66,5 & 2 & 0,2 & 282 & 33,3 & 2847 & 100,0 & \\
\hline & Amarela & 24 & 55,8 & - & - & 19 & 44,2 & 43 & 100,0 & \\
\hline & Parda & 1454 & 64,2 & 5 & 0,2 & 805 & 35,6 & 2264 & 100,0 & \\
\hline & Indígena & 3 & 33,3 & - & - & 6 & 66,7 & 9 & 100,0 & \\
\hline & Ignorado & 4862 & 89,6 & 268 & 4,9 & 298 & 5,5 & 5428 & 100,0 & \\
\hline \multirow{6}{*}{$\begin{array}{l}\text { Escolaridade (em } \\
\text { anos de estudos } \\
\text { concluídos) }\end{array}$} & Nenhuma & 360 & 79,1 & 1 & 0,2 & 94 & 20,7 & 455 & 100,0 & 0,000 \\
\hline & 1 a 3 & 1541 & 93,2 & 12 & 0,7 & 101 & 6,1 & 1654 & 100,0 & \\
\hline & 4 a 7 & 1480 & 76,2 & 3 & 0,2 & 460 & 23,7 & 1943 & 100,0 & \\
\hline & 8 a 11 & 1244 & 75,6 & 3 & 0,2 & 398 & 24,2 & 1644 & 100,0 & \\
\hline & 12 ou mais & 783 & 72,1 & 7 & 0,6 & 296 & 27,3 & 1086 & 100,0 & \\
\hline & Ignorado & 5469 & 73,7 & 258 & 3,5 & 1695 & 22,8 & 7422 & 100,0 & \\
\hline \multirow{7}{*}{$\begin{array}{l}\text { Faixa etária (em } \\
\text { anos) }\end{array}$} & 13 a 19 & 194 & 58,1 & 4 & 1,2 & 136 & 4,5 & 334 & 100,0 & 0,000 \\
\hline & 20 a 29 & 2902 & 69,2 & 80 & 1,9 & 1212 & 39,8 & 4194 & 100,0 & \\
\hline & 30 a 39 & 4407 & 79,5 & 102 & 1,8 & 1033 & 33,9 & 5542 & 100,0 & \\
\hline & 40 a 49 & 2271 & 83,2 & 56 & 2,1 & 401 & 13,2 & 2728 & 100,0 & \\
\hline & 50 a 59 & 787 & 79,2 & 19 & 1,9 & 188 & 6,2 & 994 & 100,0 & \\
\hline & 60 e mais & 266 & 78,5 & 8 & 2,4 & 65 & 2,1 & 339 & 100,0 & \\
\hline & Sem D/N & 49 & 67,1 & 15 & 20,5 & 9 & 0,3 & 73 & 100,0 & \\
\hline \multirow[t]{6}{*}{ Prática sexual } & $\mathrm{HSH}^{* * * *}$ & 2307 & 77,5 & 56 & 1,9 & 615 & 20,7 & 2978 & 100,0 & 0,000 \\
\hline & $\operatorname{MSM}^{* * * *}$ & 25 & 78,1 & 2 & 6,3 & 5 & 15,6 & 32 & 100,0 & \\
\hline & Heterossexual & 6311 & 76,3 & 171 & 2,1 & 1792 & 21,7 & 8274 & 100,0 & \\
\hline & Bissexual & 875 & 76,3 & 22 & 1,9 & 250 & 21,8 & 1147 & 100,0 & \\
\hline & Não se aplica & 147 & 82,1 & 3 & 1,7 & 29 & 16,2 & 179 & 100,0 & \\
\hline & Ignorado & 1211 & 76,0 & 30 & 1,9 & 353 & 22,1 & 1594 & 100,0 & \\
\hline \multirow{3}{*}{$\begin{array}{l}\text { Exposição } \\
\text { sanguínea }^{* *}\end{array}$} & Sim & 2497 & 92,0 & 21 & 0,8 & 196 & 7,2 & 2714 & 100,0 & 0,000 \\
\hline & Não & 3971 & 66,4 & 10 & 0,2 & 1996 & 33,4 & 5977 & 100,0 & \\
\hline & Ignorado & 4408 & 80,0 & 253 & 4,6 & 852 & 15,5 & 5513 & 100,0 & \\
\hline Total $^{* * *}$ & & 10876 & 76,6 & 284 & 2,0 & 3044 & 21,4 & 14204 & 100,0 & \\
\hline
\end{tabular}

${ }^{*}$ p-valor obtido do Teste Qui-Quadrado com correção de Yates e exato de Fisher, sem o critério Descartado. O Teste Qui-Quadrado não considerou as variáveis com zero e não informados. ${ }^{* *}$ Foram considerados para exposição sanguínea: UDI, antecedente de hemofilia, transfusão sanguínea e acidente com material biológico. ${ }^{* *}$ Todas as porcentagens são relacionadas com os valores correspondentes de cada linha das variáveis. ${ }^{* * * *} \mathrm{HSH}$ (homens que fazem sexo com homens), MSM (mulheres que fazem sexo com mulheres).

Houve aumento significativo nos coeficientes de incidência por aids, no período de 1983 a 1989, como grande variação anual percentual, de $108,5 \%$ ao ano (IC: $90,3-128,4$ ), enquanto que no período seguinte o aumento anual foi menor, e $13,2 \%$ ao ano (IC: $8,2-18,3$ ). Para o período de 1998 a 2016 houve redução da incidência, com variação anual percentual de $-6,2 \%$ ao ano (IC: $-7,4--4,9)$ (Tabela 2).

A regressão segmentada considerada mais adequada para ajustar os coeficientes de incidência no critério de notificação aids em maiores de
13 anos em Campinas foi aquela com três pontos de mudança, nos anos de 1983, 1991 e 1998, ou seja, foram suficientes quatro segmentos de reta para representar a tendência do coeficiente no período de 1980 a 2016 (Figura 2).

\section{Discussão}

A tendência dos coeficientes de incidência de aids (todos os critérios) apresentou três pontos de mudança no período, com grande aumento 
A

140

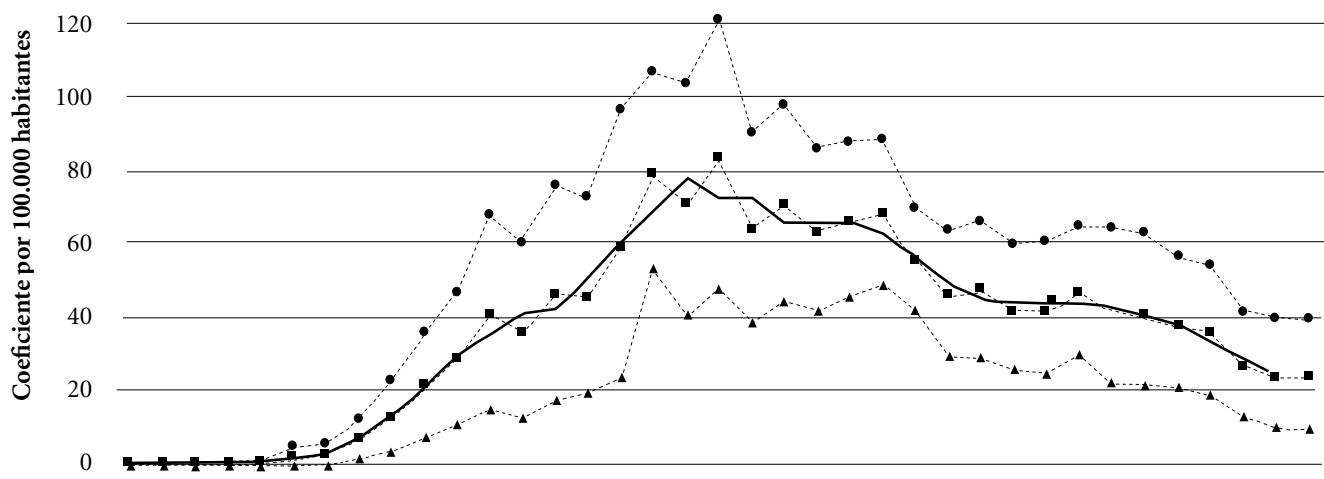

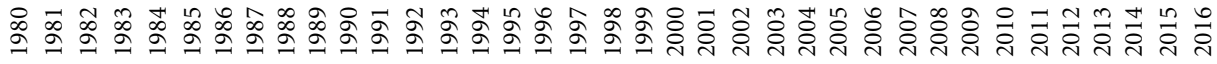

…… Masculino … Feminino … $\square$ Total $\longrightarrow$ Total (suavizada)
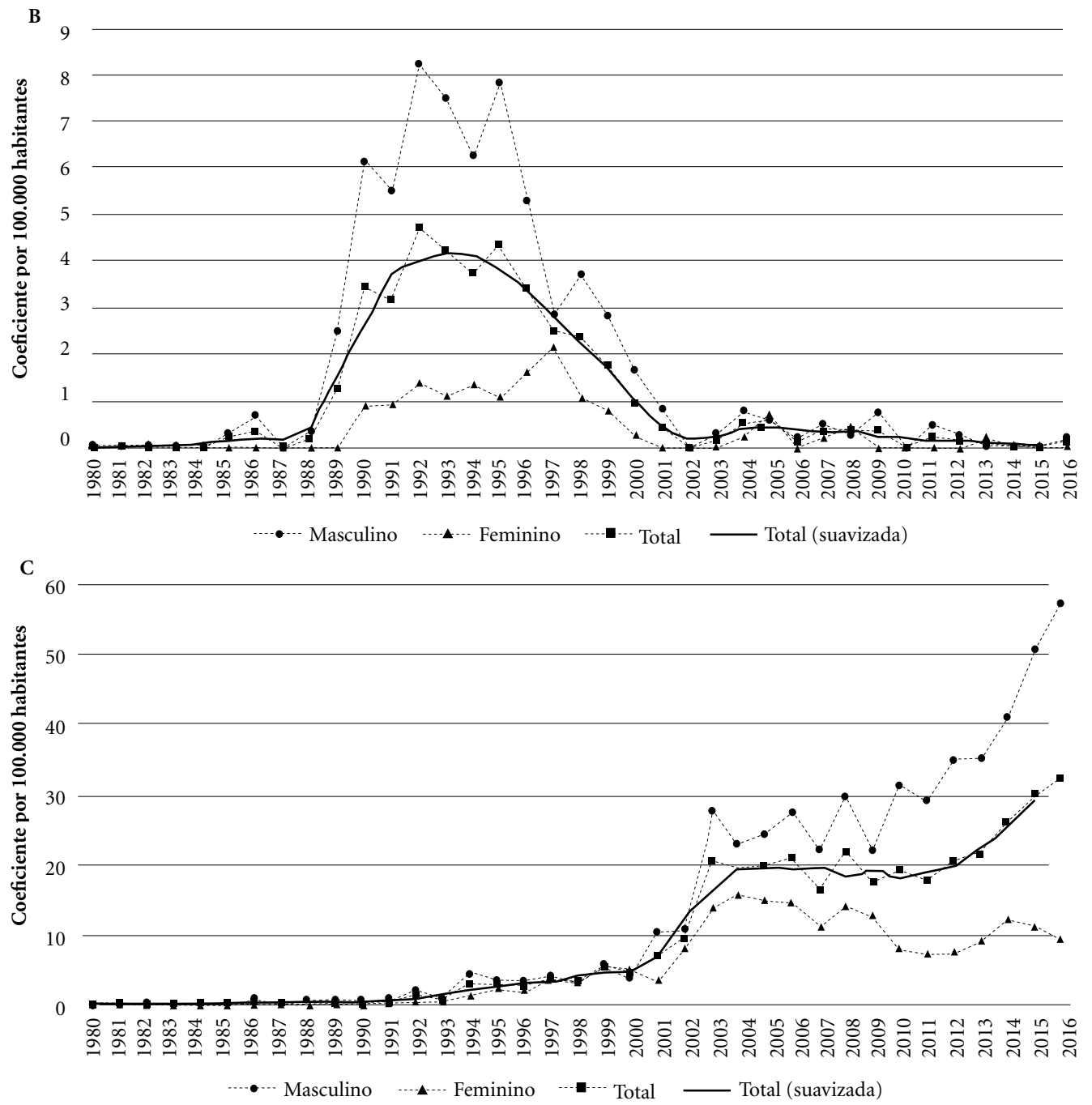

Figura 1. Coeficientes de Incidência (por 100.00 habitantes) associados à aids (CID 10: B.20 a B.24) em indivíduos maiores de 13 anos segundo critério diagnóstico aids CDC (A), Óbito (B) e HIV+ (C) e sexo (o total das notificações possui série suavizada por médias móveis de três pontos). Campinas-SP, Brasil, 1980-2016. 
do risco 1983-1989; diminuição da incidência a partir de 1998; e inversão da tendência para queda após 1999 até 2016.

As notificações por critério óbito apresentaram um período delimitado de alta incidência entre 1988 e 2002, e os registros de infecção pelo HIV por 100 mil habitantes foram crescentes a partir de 1993 em Campinas, mais elevada entre os homens. As mudanças adotadas nos critérios de notificação ao longo período em questão não trouxeram implicações para a análise temporal apresentada, uma vez que simplificaram as definições da infecção e da doença ${ }^{1,2}$.

Tabela 2. Variação anual percentual dos coeficientes de incidência por aids (critério 100) obtidas pela Joinpoint Regression. Campinas-SP, Brasil, 1980-2016.

\begin{tabular}{rrr}
\hline Período & APC $^{*}$ \%) & \multicolumn{1}{c}{ IC(95\%) } \\
\hline 1980 a 1983 & 17,7 & $-4,0-44,3$ \\
1983 a 1989 & 108,5 & $90,3-128,4$ \\
1989 a 1998 & 13,2 & $8,2-18,3$ \\
1998 a 2016 & $-6,2$ & $-7,4--4,9$
\end{tabular}

*APC: Annual Percentual Change (Variação Anual Percentual), $\mathrm{p}<0,05$.
A diminuição da velocidade de crescimento da incidência de aids na década de 90 coincide com a implantação em Campinas do Ambulatório Municipal de DST/Aids em 1990, do Programa Municipal de Doenças Sexualmente Transmissíveis e Aids e do primeiro Centro de Orientação e Apoio Sorológico, ambos em $1993^{13}$. Estes centros assumiram a assistência e as ações de prevenção, testagem do HIV e aconselhamento, conforme preconizava o Ministério da Saúde.

A queda dos coeficientes de incidência a partir de 1998 possivelmente foi reflexo das ações do Programa de DST-Aids, do diagnóstico precoce e da dispensação de terapia antirretroviral combinada aos pacientes com aids. O Centro de Referência (CR) em Infecções Sexualmente Transmissíveis (IST)/Aids foi criado em 2002, agregando o ambulatório, o centro de aconselhamento e $\mathrm{o}$ atendimento domiciliar terapêutico, que existia desde 1996.

$\mathrm{O}$ acesso gratuito e universal ao tratamento com antirretrovirais no Sistema Único de Saúde, associado com recomendações técnicas e protocolos (Lei que dispõe sobre a distribuição gratuita de medicamentos aos portadores do HIV e doentes de Aids no 9313 de 1996) foram estratégias do Ministério da Saúde que influenciaram diretamente a incidência e a mortalidade devido

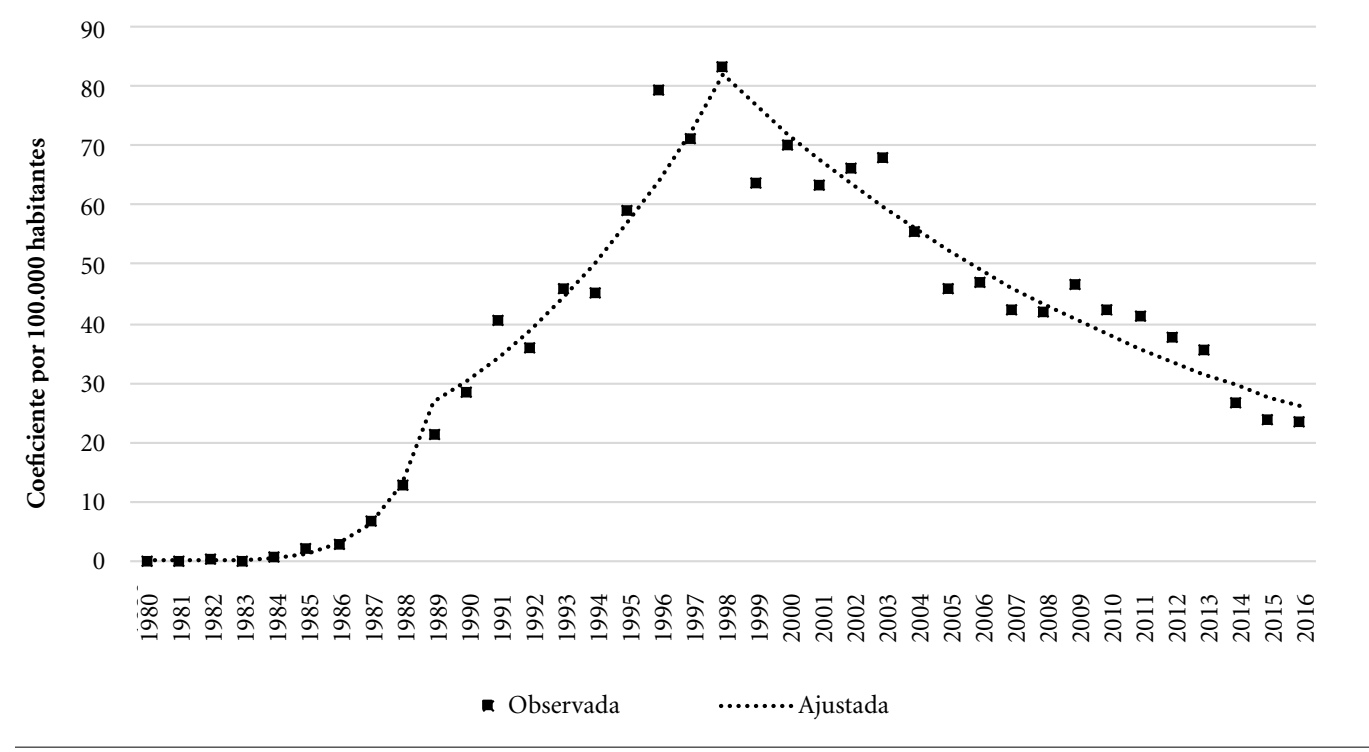

Figura 2. Coeficientes de Incidência por 100.000 habitantes em maiores de 13 anos associados à aids (CID 10: B.20 a B.24 - critério CDC) segundo ano de diagnóstico, obtidos com regressão segmentada (Joinpoint Regression). Campinas-SP, Brasil, 1980-2016. 
à aids em várias regiões do Brasil ${ }^{10}$. $\mathrm{O}$ aumento do percentual de pacientes com carga viral indetectável ou reduzida possivelmente teve impacto na diminuição de novos $\operatorname{casos}^{19}$. Nesta perspectiva, esta política pública contribui para reduzir desigualdades na saúde, não só em Campinas ${ }^{20}$.

Notam-se diferentes padrões epidemiológicos nos três períodos identificados pela análise Joinpoint Regression. Em todo o período, a maioria dos casos ocorreu em homens, embora o teste HIV tenha sido incorporado na rotina do pré-natal, sugerindo maior oportunidade de detecção na população feminina. A velocidade de detecção de novos casos diminuiu gradativamente nos anos 90, apesar do aumento do número absoluto de casos até 1998 e de mudanças no perfil sociodemográfico da epidemia. O decréscimo percebido no final da série estudada pode não refletir a real incidência devido à possível inclusão de novos casos retroalimentados nos bancos de dados oficiais ${ }^{21}$.

O aumento das taxas de notificação de pacientes com HIV em Campinas foi perceptível desde 1993 com o maior incremento a partir de 2000. A implantação do Programa municipal em 1993 e do CR em IST-aids antecipou-se à estratégia do Ministério da Saúde que instituiu a notificação compulsória dos pacientes com HIV a partir de 2014 (Portaria MS No1.271, 2014). Até então realizada somente nos serviços sentinela de atendimento às infecções sexualmente transmissíveis e aids.

O aumento das notificações da infecção por HIV é de difícil interpretação devido a mudanças na disponibilidade dos exames, na disposição dos profissionais de saúde, dos indivíduos para serem testados, na organização de campanhas específicas além de diferenças entre os sistemas de notificação adotados pelo estado de São Paulo e pelo Ministério da Saúde. A representatividade destas taxas muda diante do longo período de latência até a imunodepressão. Entretanto estudos no Brasil e outros países chamam a atenção para o crescimento da infecção pelo HIV em HSH e populações vulneráveis ${ }^{9,21}$. Vale destacar a importância de garantir não somente o acesso ao tratamento, mas também à prevenção.

Por outro lado, registra-se a elevação da incidência de aids pelo critério óbito no município de 1989 a 2001, com pico no início dos anos 90 e queda brusca a partir de 1996 em homens e $1997 \mathrm{em}$ mulheres. Trata-se de um indicador que revela radicalmente o atraso no diagnóstico e o caráter silencioso da epidemia, pois a infecção foi notificada a partir do óbito. A queda dessas taxas provavelmente deveu-se ao crescente acesso da população aos serviços de prevenção, assistência e detecção precoce da infecção por HIV no município, aumentado as chances de sobrevivência dos pacientes, mesmo diagnosticados em fase de intensa imunodepressão $0^{14}$.

Esses aspectos se relacionam fortemente com a redução da notificação de casos com o critério óbito, pois a partir da detecção, acompanhamento e tratamento da doença, novos casos passaram a ser descobertos precocemente e com melhor prognóstico que em épocas anteriores ${ }^{22}$.

A inclusão do teste HIV no Pré-natal (Portaria MS no 2104, 2002) ampliou o diagnóstico precoce de indivíduos com HIV, resultando na investigação também de parceiros e familiares, além da prevenção da transmissão vertical. Ao mesmo tempo, a assistência do pré-natal e do parto são uma forma viável de obter estimativas de prevalência da infecção por HIV na população de mulheres saudáveis e sexualmente ativas, servindo, com algumas restrições, como indicadores sentinela da infecção em países em desenvolvimento $^{23,24}$.

A redução do critério "óbito" de notificação e o aumento nas taxas de notificação da infecção pelo HIV sugerem que as pessoas possuem acesso mais precoce ao diagnóstico. Por outro lado, o Ministério da Saúde disponibiliza teste rápido para HIV e campanhas de testagem com parceria entre estados, municípios e sociedade civil ${ }^{25}$.

$\mathrm{O}$ fato do número de casos confirmados de HIV ser menor entre HSH do que entre os heterossexuais em Campinas reflete o recrudescimento da epidemia na população de $\mathrm{HSH}$, que era majoritária entre os casos de doença no início da epidemia, porém teve sua participação proporcional progressivamente reduzida e vem mostrando novo acréscimo nos últimos anos, fato que encontra paralelos na literatura médica nacional e internacional. Dados recentes da epidemia no país revelam aumento da incidência de HIV em homens, HSH, com baixa escolaridade, e adesão precária a medicação ${ }^{26,27}$. Aumento também observado em outros países ${ }^{28,29}$. Na Suíça, o aumento da transmissão do HIV entre os HSH refletiu desconhecimento do seu estado de infecção e comportamento sexual de risco contínuo $(\mathrm{CSRC})^{30}$.

Apesar de campanhas de acesso à informação, a despreocupação com a infecção, a ideia de que aids é uma doença com tratamento e o CSRC passam a ser questões a serem retomadas por atividades educativas específicas ${ }^{31}$. Estudo nacional realizado em 2008-2009 com HSH residentes em 
dez cidades brasileiras verificou que ainda é elevado o número de indivíduos com baixo conhecimento sobre HIV/aids e alta vulnerabilidade social $^{32}$.

A ampliação de estratégias de testagem e o tratamento precoce dos indivíduos com HIV podem reduzir a incidência da doença na população ${ }^{33}$. O Ministério da Saúde do Brasil desde 2013, adota como diretriz nacional, o tratamento com antirretrovirais de todos os indivíduos com o diagnóstico de infecção pelo HIV ${ }^{34}$. Destaca-se a proposição da Organização Mundial da Saúde (OMS) da "Vigilância do HIV de segunda geração" a qual, além da infecção por HIV, acrescenta a importância de se antecipar ao diagnóstico da ocorrência da doença, identificando grupos de pessoas com comportamentos que os expõem à infecção pelo $\mathrm{HIV}^{35}$.

A técnica de regressão segmentada neste estudo identificou pontos de mudança significativos da tendência da detecção da aids, uma vez que estimou a variação percentual anual. Complementar a essa técnica, a suavização por médias móveis revelou mais claramente o comportamento da incidência segundo os critérios de notificação e possíveis pontos de mudança na série temporal, já a RS identificou tais pontos, bem como estimou os incrementos em cada segmento da reta ${ }^{36}$. Embora esses pontos de mudança que nem sempre correspondem aos reais pontos de variação ${ }^{36}$, estas técnicas podem ser incorporadas às análises dos dados epidemiológicos dos serviços no país uma vez que visualizam de forma mais ampla como as incidências se comportaram na série histórica estudada.

Entre as limitações deste estudo, ressaltamse a utilização de dados secundários e o grande percentual de informações faltantes (categoria "ignorados"), particularmente, como é de se esperar, entre os casos notificados com critério “óbito" e na variável de exposição sanguínea, dificultando o estudo do perfil destes pacientes nos vários períodos da epidemia no município. Os dados ignorados quanto à escolaridade são, em número, muito maiores que os esperados, o que indica que PVHA ainda estão em acompanhamento da rede de saúde e tal dado não consta em suas notificações.

Por outro lado, a cobertura dos registros de casos de aids no SINAN na última década foi crescente com melhora da qualidade das notificações. Os casos de aids com critério óbito após a notificação inicial, são revistos na Fundação Sistema Estadual de Análise de Dados (Seade), São Paulo, diminuindo as imprecisões e retroalimentando o Sistema de Informação de Mortalidade (SIM) no estado. Por outro lado, a mudança no sistema de notificação de casos em 2003 dificultou a compatibilização das variáveis entre os sistemas de informação, diminuindo de nove critérios diagnósticos de aids para três. Como mencionado anteriormente, as características assistenciais são distintas entre as cidades do estado de São Paulo e tais mudanças podem ser pesquisadas a fim de nivelá-las junto a Secretaria Estadual de Saúde.

Apesar destas limitações foram analisados todos os casos de aids e HIV notificados e investigados em Campinas cobrindo os vários períodos da epidemia. Em conclusão, os resultados mostram um declínio consistente da incidência de aids (critério CDC), e também pelo critério óbito além de um aumento das notificações de infecção por HIV no período de estudo em Campinas. Embora os coeficientes de incidência ainda permaneçam altos no município, estas tendências são compatíveis com o impacto positivo das políticas de deteç̧ão precoce e acesso ao seguimento clínico e terapêutico dos indivíduos com HIV e aids na cidade.

Torna-se importante o desenvolvimento de ações e estratégias para melhorar a qualidade dos dados dos sistemas de informação e as implicações para o aprimoramento das ações de monitoramento e avaliação da caracterização dos casos de HIV/aids, visando o desenvolvimento de políticas públicas mais efetivas para o enfrentamento da infecção/doença. 


\section{Colaboradores}

MC Melo participou na concepção do estudo, análises dos dados, revisão do texto, discussão dos resultados e na escrita do manuscrito. MR Donalisio participou na concepção do estudo, coleta e análise dos dados, revisão do texto, discussão dos resultados e na escrita do manuscrito. VC Almeida participou na análise dos dados, revisão do texto, discussão dos resultados e na escrita do manuscrito.

\section{Referências}

1. Brasil. Ministério da Saúde (MS). Secretaria de Políticas de Saúde. Coordenação Nacional de DST e Aids. Revisão da definição nacional de casos de aids em indivíduos com 13 anos ou mais, para fins de vigilância epidemiológica. Brasília MS; 1998.

2. Brasil. Ministério da Saúde (MS). Secretaria de Vigilância em Saúde. Programa Nacional de DST e Aids. Boletim Epidemiológico - Aids. Brasília MS; 2003.

3. Secretaria de Estado de Saúde de São Paulo. Programa Estadual de DST/AIDS. Divisão de Vigilância Epidemiológica. Boletim Epidemiológico C.R.T. - DST/ AIDS. C.V.E. Ano III - $n$ 1. São Paulo: Secretaria de Estado de Saúde de São Paulo; 2001.

4. Secretaria de Estado de Saúde de São Paulo. Coordenadoria do Controle de Doenças. Coordenação Estadual de DST/AIDS. Divisão de Vigilância Epidemiológica. Notificação dos Casos Assintomáticos Soropositivos para o HIV no Sinan no Estado de São Paulo 2000 a 2010. Boletim Epidemiológico Paulista (Bepa) 2011; 8(95):14-21.

5. Brasil. Ministério da Saúde (MS). Relatório de Implementação e Avaliação - 1998 a 2002 - Acordo de empréstimo BIRD 4392/BR - Projeto AIDS II. Brasília: MS; 2002.

6. Brasil. Ministério da Saúde (MS). Secretaria de Vigilância em Saúde. Boletim Epidemiológico - Aids e DST. Brasília: MS; 2016.

7. Neves LAS, Reis RK, Gir E. Compliance with the treatment by patients with the co-infection HIV/tuberculosis: integrative literature review. Rev Esc Enferm USP 2010; 44(4):1135-1141.

8. Silva ACO, Reis RK, Nogueira JA, Gir E. Quality of life, clinical characteristics and treatment adherence of people living with HIV/AIDS. Rev Latino-Am Enferm 2014; 22(6):994-1000.

9. Beyer C, Baral SD, Griensven F, Goodreau SM, Chariyalertsak S, Wirtz AL, Brookmeyer R. Global epidemiology of HIV infection in men who have sex with men. Lancet 2012; 380(9839):367-377.

10. Szwarcwald CL, Pascom ARP, Souza Júnior PR. Estimation of the HIV Incidence and of the Number of People Living With HIV/AIDS in Brazil, 2012. J AIDS Clin Res 2015; 6(3):430.

11. Dourado I, Veras MASM, Barreira D, Brito AM. AIDS epidemic trends after the introduction of antiretroviral therapy in Brazil. Rev Saude Publica 2006; 40(Supll):9-17.

12. Secretaria de Estado de Saúde de São Paulo. Coordenadoria de Controle de Doenças. Centro de Vigilância Epidemiológica. Centro de Referência e Treinamento em DST/Aids - CRTDST/AIDS-SP. Programa Estadual de DST/Aids de São Paulo. Boletim Epidemiológico CRT - DST/AIDS. CVE. Ano XXXIII - $n$ 1. São Paulo: Secretaria de Estado de Saúde de São Paulo; 2017.

13. Secretaria Municipal de Saúde de Campinas. Programa Municipal de DST/Aids de Campinas. Histórico [Internet]. [acessado 19 Nov 2018]. Disponível em: http://www.saude.campinas.sp.gov.br/programas/ programa_dst_aids/programa_dst_aids.htm 
14. Laurenti R, Jorge MHPM, Lebrão ML, Gotlieb SLD. Estatísticas de Saúde. São Paulo: EPU; 1987.

15. Branch SMA, Program SR, Institute NC. Joinpoint Regression Program. June 2017.

16. Martinez-Beneito MA, García-Donato G, Salmerón D. Bayesian joipoint regression model with unknown number of break-points. Annals Applied Statistics 2011; 5(3):2150-2168

17. Kim HJ, Fay MP, Feuer EJ, Midthune DN. Permutation tests for joinpoint regression with applications to cancer rates. Stat Med 2000; 19(3):335-351.

18. Brasil. Ministério da Saúde (MS). Secretaria de Vigilância em Saúde. Coordenação-Geral de Desenvolvimento da Epidemiologia em Serviços. Guia de Vigilância em Saúde. Brasília: MS; 2017.

19. Brignol S, Dourado I, Amorim LD, Kerr LRFS. Vulnerability in the context of HIV and syphilis infection in a population of men who have sex with men (MSM) in Salvador, Bahia State, Brazil. Cad Saude Publica 2015; 31(5):1035-1048.

20. Antunes JL, Waldman EA, Borrell C. Is it possible to reduce AIDS deaths without reinforcing socioeconomic inequalities in health? Int J Epidemiol 2005; 34(3):586-592

21. Kerr L, Kendall C, Guimarães MDC, Salani Mota R, Veras MA, Dourado I, et al. HIV prevalence among men who have sex with men in Brazil: results of the 2nd national survey using respondent-driven sampling. Medicine (Baltimore) 2018; 97(Supl. 1):S9-S15.

22. Santos NJS, Tayra A, Silva SR, Buchalla CM, Laurenti R. A aids no Estado de São Paulo. As mudanças no perfil da epidemia e perspectivas da vigilância epidemiológica. Rev Bras Epidemiol 2002; 5(3):286-310.

23. Eaton JW, Rehle TM, Jooste S, Nkambule R, Kim AA, Mahy M, Hallett TB. Recent HIV prevalence trends among pregnant women and all women in sub-Saharan Africa: implications for HIV estimates. AIDS 2014; 28(Supl. 4):S507-S514.

24. Kiarie J, Nduati R, Koigi K, Musia J, John G. HIV-1 testing in pregnancy: acceptability and correlates of return for test results. AIDS 2000; 14(10):1468-1470.

25. Abati PAM, Segurado AC. Testagem anti-HIV e estadio clínico na admissão de indivíduos em serviço de saúde especializado. Pará, Brasil. Rev Saúde Pública 2015; 49:16.

26. Szwarcwald CL, Ferreira Júnior OC, Brito AM, Luhm KR, Ribeiro CEL, Silva AM, Cavalcanti AMS, Ito TS, Raboni SM, Souza Júnior PRB, Pereira GFM. Estimation of HIV incidence in two Brazilian municipalities, 2013. Rev Saude Publica 2016; 50:55.

27. Silva JAG, Brito AM. Factors associated with non-adherence to antiretroviral therapy in adults with AIDS in the first six months of treatment in Salvador, Bahia State, Brazil. Cad Saude Publica 2015; 31(6):11881198.

28. Beyrer C, Sullivan P, Sanchez J, Baral SD, Collins C, Wirtz AL, Altman D, Trapence G, Mayer K. The increase in global HIV epidemics in MSM. AIDS 2013; 27(17):2665-2678.
29. Lippman SA, Veloso VG, Buchbinder S, Fernandes NM, Terto V, Sullivan PS, Grinsztejn B. Over-thecounter human immunodeficiency virus self-test kits: time to explore their use for men who have sex with men in Brazil. Braz J Infect Dis 2014; 18(3):239-244.

30. van Sighem A, Vidondo B, Glass TR, Bucher HC, Vernazza P, Gebhardt M, de Wolf F, Derendinger S, Jeannin A, Bezemer D, Fraser C, Low N, Swiss HIV Cohort Study. Resurgence of HIV infection among men who have sex with men in Switzerland: mathematical modelling study. PLoS One 2012; 7(9):e44819.

31. Mayer KH, Mimiaga MJ. Past as prologue: the refractory and evolving HIV epidemic among men who have sex with men. Clin Infect Dis 2011; 52(11):13711373.

32. Gomes RRFM, Ceccato MGB, Kerr LRFS, Guimarães MDC. Factors associated with low knowledge on HIV/AIDS among men who have sex with men in Brazil. Cad Saude Publica 2017; 33(10):e00125515.

33. Das M, Chu PL, Santos GM, Scheer S, Vittinghoff E, McFarland W, Colfax GN. Decreases in community viral load are accompanied by reductions in new HIV infections in San Francisco. PLoS One 2010; 5(6):e11068.

34. Brasil. Ministério da Saúde (MS). Secretaria de Ciência, Tecnologia e Insumos Estratégicos. Protocolo Clínico e Diretrizes Terapêuticas para Manejo da infeç̧ão pelo HIV em adultos. Brasília: MS; 2017.

35. World Health Organization (WHO). Departamento Enfermedades Transmisibles Vigilancia y Respuesta. Vigilancia del VIH de segunda generación. El próximo decênio. Genebra: WHO; 2000

36. Melo MC, Ferraz RO, Nascimento JL, Donalisio MR. Incidence and mortality of children and teenagers with AIDS: challenges in the southern region of Brazil. Cien Saude Colet 2016; 21(12):3889-3898.

Artigo apresentado em 17/08/2018

Aprovado em 13/04/2019

Versão final apresentada em 15/04/2019

Editores chefes: Romeu Gomes, Antônio Augusto Moura da Silva 
